\title{
Flomax - Important - be Aware!
}

\author{
Charles (Chuck) Maack* \\ Prostate Cancer Advocate/Activist, USA \\ *Corresponding author: Charles (Chuck) Maack - Prostate Cancer Advocate/Activist Wichita, Kansas Chapter, Us TOO Intl., Inc. \\ 8201 East Harry St., Ste 1804 Wichita, KS 67207, USA
}

To Cite This Article: Charles (Chuck) Maack. Flomax - Important - be Aware!. Am J Biomed Sci \& Res. 2021 - 13(6). AJBSR.MS.ID.001923. DOI: 10.34297/AJBSR.2021.13.001923.

Received: 眥 July 26, 2021; Published: 眥 August 10, 2021

\section{Opinion}

\section{Disclaimer}

Please recognize that I am not a Medical Doctor. I have been an avid student researching and studying prostate cancer as a survivor and continuing patient since 1992. I have dedicated my retirement years to continued research and study in order to serve as an advocate for prostate cancer awareness, and, from a activist patient's viewpoint, to help patients, caregivers, and others interested develop an understanding of prostate cancer, its treatment options, and the treatment of the side effects that often accompany treatment. Readers of this paper must understand that the comments or recommendations I make are not intended to be the procedure to blindly follow; rather, they are to be reviewed as my opinion, then used for further personal research, study, and subsequent discussion with the medical professional/physician providing prostate cancer care.

Many men dealing with prostate cancer or Benign Prostatic Hyperplasia (BPH) - and in this case continence/incontinence issues - are prescribed the alpha blocker Flomax (tamsulosin hydrochloride). And among these men are those who find they require cataract surgery and have the procedure performed. Come to find out, some men, particularly those over 65, taking Flomax or other alpha blockers are experiencing cataract surgery complications. For this reason, the report explained in the below URL recommends that if possible, alpha blocker medications should be discontinued prior to eye surgery in older patients (Note:

You may have to subscribe to URO Today to open....information to do so is at the end of this paper)

http://tinyurl.com/kn8nej3
Physician Luis Garcia-Buneul provided important information when a patient experienced a stroke while taking Flomax at the same time as taking Viagra. Flomax is an alpha-blocker and causes vasodilatation. The combination of Flomax and Viagra can cause a similar blood-pressure to drop that can occur with nitroglycerin. In the milder reactions, it presents as "postural hypotension"; just getting dizzy or fainting when getting upright. In the more severe reactions, the blood flow to the brain gets so restricted that some small areas can be oxygen deprived to the point of permanent damage (stroke). Although drug warnings about using Viagra in conjunction with nitrates or alpha-blockers are given, I don't think that enough emphasis is being placed by those warnings on the more serious consequences of such combinations.

And yet more from Dr. Garcia-Buneul to a patient taking both Flomax and medicine for his heart: "The combination of Flomax and isosorbide mononitrate was most likely to blame for your problems. The mononitrate compound is good for angina because it causes dilatation of the heart's coronary arteries (The pain in angina is because not enough blood is flowing through the coronary arteries). The combination of two vasodilators proved in your case to be a dangerous one. You say: "I felt as if all the blood was draining away from my body, my face taking turning deathly white". In a way, your blood was draining away from your body because when your arteries dilate in large scale, it is as if you would have lost large amounts of blood until the arteries return to their normal diameter. With massive vasodilatation, the brain is the first to feel it, in the form of dizziness, fainting or even thrombosis (stroke). Lying down as you did helps because more blood can flow to your brain in that position." 
Retired Ophthalmologist Richard Chenoweth, a prostate cancer patient, provided this very important information: "The "floppy iris" syndrome in patients on Flomax (an alpha-blocker) is well known to ophthalmologists doing cataract surgery. After installing an intraocular lens, constricting the pupil is part of the procedure, since it stabilizes the lens and vitreous behind it, so that the surgeon can then irrigate out the viscous material that is put into the anterior chamber of the eye in order to better slide the intraocular lens into place. Irrigating out this material is necessary in order to prevent postoperative glaucoma. When men take Flomax for urinary retention due to $\mathrm{BPH}$, the usual miotic agents to constrict the pupil don't work. The Flomax ads now state that "you must let your eye doctor know you are on Flomax if you are having cataract surgery". If the cataract surgeon knows the patient is on Flomax, there are alternatives that the surgeon can use that will work during surgery. The obvious solution is to have the patient discontinue Flomax before cataract surgery. I was not aware that this was a particular problem in patients over 65 years of age- I don't know why age would accentuate it, other than most patients taking Flomax are probably over 65 years of age (as are most of those undergoing cataract surgery). A problem with Flomax is that it is only available in sustained release capsules in a relatively large dose; the capsule can't be cut in half to reduce the dose. I couldn't tolerate Flomax for this reason, because it limited my ability to get my heart rate and cardiac output up to climb hills after taking it (I am an active hiker and hill climber). This is a common problem with active seniors who try Flomax. I take Hytrin (initially 2 mgm, now $4 \mathrm{mgm}$ ) at night with success. It reduces the number of times I need to get up to pee at night, and no adverse effects when I go hiking. Hytrin is also sold in sustained release capsules: but, it can be obtained in tablets as low as $1 \mathrm{mgm}$. My 92 year old uncle (who has never had any prostate surgery, biopsies, or PSA's) does well with $1 \mathrm{mgm}$ of Hytrin at night. If he tries to take $2 \mathrm{mgms}$, he gets orthostatic hypotension (gets weak and dizzy due to low blood pressure when he sits up). He could never tolerate Flomax-it would be dangerous, and likely cause fainting or worse!"

\section{Here is an Interesting Read}

http://www.cvpharmacology.com/vasodilator/vasodilators. htm

And more: The pharmacokinetic interaction between cimetidine and FLOMAX capsules was investigated. The results indicate significant changes in tamsulosin HCI clearance $(26 \%$ decrease) and AUC (44\% increase). Therefore, FLOMAX capsules should be used with caution in combination with cimetidine, particularly at doses higher than $0.4 \mathrm{mg}$.

\section{Here are Other Side Effects that are Possible}

a) Abnormal ejaculation -- occurring in up to 18.1 percent of people (look up

b) Flomax Sexual Side Effects)

c) Runny or stuffy nose -- in up to 17.9 percent

d) Dizziness -- in up to 17.1 percent

e) Infections, including the common cold or flu -- in up to 10.8 percent

f) General body pain -- in up to 8.5 percent

g) Back pain -- in up to 8.3 percent

h) Diarrhea -- in up to 6.2 percent

i) Sore throat -- in up to 5.8 percent.

There are Several Less Common Side Effects of Flomax that Occurred in 1 to 5 Percent of People
a) Increased coughing
b) Sleepiness
c) Chest pain
d) Upset stomach (nausea)
e) Sinus infection (sinusitis)
f) Decreased sex drive (libido)
g) Blurred vision
h) Tooth problems
i) Trouble sleeping (insomnia)
j) Spinning sensation (vertigo).

And finally, it is IMPORTANT to read and heed:

\section{FDA Warning/Regulatory Alert}

Note from the National Guideline Clearinghouse: This guideline references a drug(s) for which important revised regulatory and/or warning information has been released.

On November 22, 2005, Boehringer Ingelheim and the U.S. Food and Drug Administration (FDA) notified healthcare professionals of revisions to PRECAUTIONS and ADVERSE REACTIONS sections of the prescribing

information for Flomax, indicated for the treatment of the signs and symptoms of benign prostatic hyperplasia (BPH). A surgical 
condition termed Intraoperative Floppy Iris Syndrome (IFIS) has been observed during phacoemulsification cataract surgery in some patients treated with alpha-1 blockers including Flomax. Most of these reports were in patients taking the alpha-1 blocker when IFIS occurred, but in some cases alpha-1 blocker had been stopped prior to surgery. It is recommended that male patients being considered for cataract surgery, as part of their medical history, be specifically questioned to ascertain whether they have taken Flomax or other alpha-1 blockers. If so, the patient's ophthalmologist should be prepared for possible modifications to their surgical technique that may be warranted should IFIS be observed during the procedure. See the FDA Web site for more information.

\section{Subscribing to URO TODAY}

If not a subscriber to URO TODAY newsletters and you click on a URO TODAY URL to read one of their papers, you will likely be asked to register. Or, if you want to subscribe anyway, go to http://www. urotoday.com/. When you do, follow these directions and once subscribed you will gain access to the abstracts of most all their papers and future URO TODAY daily newsletters:

For subscribing, fill out usual information and provide a password you will use if needed to access URO TODAY, then for the following click as I note:
Profession: Patient/Consumer

Are you: Other

What is your specialty: Other

What industry do you work in: Other

(The Profession and "Other" above if you are not already a medical professional)

Then make your choice of the format you want to receive the newsletters (Plain? HTML?)

Then where it asks the newsletter to which you want to subscribe, click on "Uro Alert - Prostate Cancer Daily"

Then scroll down following other directions before clicking on "Register"

You will then receive their daily newsletters/abstract papers regarding prostate cancer, and when you click on any subject in those newsletters, they will automatically open to the abstract - or they will ask for your email address and password, which will then open the abstract. 